\title{
Le contrat source d'obligations
}

\author{
Alain BÉRY
}

Adresse

de correspondance :

Alain BERY

9 , rue Vézelay

75008 Paris
Le principe juridique traditionnel médecin-malade repose, sauf exceptions, sur l'existence d'un contrat ${ }^{1}$, comme l'a affirmé l'arrêt de principe du 20 mai $1936^{2}$. Avant cette date, la relation médecin - malade était considérée comme une simple rencontre entre deux particuliers et sanctionnée en références aux articles $1382^{3}$ et $1384^{4}$ du Code civil.

Le contrat médical est un contrat civil, synallagmatique (à la qualification d'ailleurs incertaine ${ }^{5,6}$ ) régi par la liberté contractuelle et l'autonomie de volonté ; il est formé ${ }^{7}$ par l'échange des consentements, et le contenu des obligations est déterminé par les parties. La liberté contractuelle implique le choix $\mathrm{du}$ contractant, et cette règle civile rejoint un des principes fondamentaux de la médecine : le libre choix du médecin par le patient. Ce principe constitue une garantie pour le malade, un symbole et un témoignage de 1'humanisme médical essentiel aux rapports de confiance ${ }^{8,9}$.

Dans le contrat médical traditionnel, le choix du médecin est d'autant plus libre qu'il est effectué généralement pour un acte, une intervention, et le contrat prend fin à son terme normal (sauf restrictions du L. 1111-2). Ce contrat est consensuel et n'est donc soumis à aucune forme ; mais le principe du consensualisme tend à disparaittre dans les nouvelles relations ${ }^{10}$. Dans le classique contrat médical, l'objet de la prestation des contractants, c'est-à- dire «ce à quoi le débiteur est tenu envers le créancier ${ }^{1 l}$ " est déterminé par les parties. Il s'agit pour un médecin d'assurer une prestation de soins dans le respect des règles déontologiques et, éventuellement, conventionnelles, et pour le patient de rémunérer ${ }^{12}$ cette prestation ${ }^{13}$.

Le contrat médical semble appartenir à la classe $d u$ «contrat d'entreprise par lequel une personne s'engage moyennant rémunération à accomplir de manière indépendante un travail au profit d'une autre sans la représentery (Art. 1792-5 C. civ.). Mais attention, l'entrepreneur est tenu à une obligation de résultat alors que l'odontologiste est soumis à une obligation de moyens (sauf en ce qui concerne l'élément prothétique qui est assujetti à une obligation de résultat ${ }^{14,15}$ ).

Défini par l'arrêt de la Cour de cassation du 14 mars $1967^{16}$, «Le contrat entraîne l'obligation pour le premier de donner au second des soins conformes aux règles consacrées par la pratique dentaire et aux données de la science. Le praticien est responsable des suites dommageables des soins, si eu égard à cette obligation de moyens, il s'est rendu coupable d'une imprudence, d'une inattention ou d'une négligence révélant la méconnaissance de ses devoirs).

Toute inexécution d'une obligation contractuelle constitue une faute à moins que le débiteur défaillant ne parvienne à prouver l'existence d'une cause étrangère. 
Le droit de la responsabilité médicale ${ }^{17}$ est un droit principalement prétorien qui repose sur des équilibres fondamentaux. Praticien d'une science essentiellement aléatoire, le médecin a vu tout d'abord sa responsabilité juridique limitée au principe de l'obligation de moyens, ce qui exclut toute responsabilité en dehors d'une faute prouvée ${ }^{18}$. On a voulu éviter que l'acte médical ne devienne comme d'habitude une tendance à une fin, sans volonté ni conscience ${ }^{19}$ : la volonté qui suppose intelligence et réflexion, doit inciter le médecin à ne poursuivre l'exécution d'un acte que s'il s'en représente les conséquences et s'il est capable d'adopter à son encontre une attitude critique. L'homme demande de plus en plus souvent à la science un verdict sur sa destinée. Il faut éclairer l'homme dans la mesure où, physiquement et psychologiquement, il peut l'être, sans pour autant mettre à la charge du praticien des obligations matérielles et morales démesurées ${ }^{20}$.

1. Béry A. Le contrat de soins. Collection : Odontologie Droit Éthique. Paris : SID, 1997.

2. «ll se forme entre le médecin et son client un véritable contrat comportant, pour le praticien, l'engagement sinon bien évidemment de guérir le malade du moins de lui donner des soins non pas quelconques, mais consciencieux, attentifs, et réserve faite des circonstances exceptionnelles, conformes aux données acquises de la science; que la violation involontaire de cette obligation est sanctionnée par une responsabilité de même nature, également contractuelle».

3. «Tout fait quelconque de l'homme qui a causé à autrui un dommage, oblige celui par la faute duquel il est arrivé à la réparer».

4. «Chacun est responsable đu dommage qu'il a causé non seulement par son fait, mais encore par sa négligence ou son imprudence».

5. Harichaux M. Vers un tournant de la médecine libérale en France ? RD sanit. soc., 1994, p. 226.

6. Mémeteau G. Le droit médical. Paris : Litec, $1985, \mathrm{n}^{\circ} 24$.

7. Béry A. Conditions requises pour la formation du contrat de soins. Rev Orthop Dento Faciale 1992; 26:24-30.

8. Savatier R. et J., Auby J.-M., Péquinot H. Traité de droit médical. Paris : Lib. Techniques, 1956, n 255.

9. Gouesse E. Consentement, aléa thérapeutique et responsabilité médicale. Gaz. Pal., 16-17 juin 1999, p. 54 .

10. Harichaux M. Droit médical et hospitalier, fasc. $81, \mathrm{n}^{\circ} 73$.

11. C. civ. art. 1108.

12. Béry A. Les honoraires. Rev Orthop Dento Faciale 1993;27:27-31.

13. Harichaux M. Contrat médical et option conventionnelle, Rev. gén. dr. méd., 1999, n² 2, p. 71-89.

14. Rickmanns et Meer Van de Put. Les droits et les obligations des médecins ainsi que des dentistes, accoucheurs et infirmières, éd. Larcier Bruxelles, 1971, T. II, p. 173 : «Le dentiste contracte une obligation de résultat en fournissant un appareil de prothèse et à moins de démontrer, à l'origine du mauvais fonctionnement de ce deriier, un cas fortuit, normalement imprévisible, il répond de la qualité et de l'adaptation de l'appareil».

15. Bouvier F. Les prothèses et le droit. Gaz. Pal., 6 nov. 1993, p. 1294-1299.

16. Civ., 14 mars 1967, pourvoi $\mathrm{n}^{\circ}$ 66-10.284.

17. Béry A., Creusot G., Sapanet M. L'expertise dentaire et maxillo-faciale. Paris : Masson, 1996.

18. Petroni-Maudière N. L'institution d'une obligation de sécurité de résultat à la charge du médecin ou le risque d'une rupture des équilibres fondamentaux du droit de la responsabilité médicale. Rev, gén. dr. méd., 2001, no 6, p. 175-196.

19. Huisman D. Vergez A. Nouveau précis de philosophie, T. 1, L'action, p. 109.

20. Bouvier F. S. Jurisprudence, note sous arrêt, 1985, p. 285-286. 